\title{
Transformaciones Microestructurales en Soldaduras Disímiles de Acero Inoxidable Austenítico con Acero Inoxidable Ferrítico
}

\author{
Sara María Aguilar-Sierra ${ }^{1}$, Claudia Patricia Serna Giraldo ${ }^{1}$, Ricardo Emilio Aristizábal-Sierra ${ }^{1}$ \\ 1 Departamento de Ingeniería de Materiales, Universidad de Antioquia, Medellín, Antioquia, Colombia.
}

\author{
Recibido: 25 Set., 2014 \\ Aprobado: 16 Mar., 2015 \\ E-mail:
}

sara.aguilar@udea.edu.co (SMAS)
Este es un artículo publicado en acceso (Open Access) abierto bajo la licencia Creative Commons Attribution NonCommercial, que permite su uso, distribución y reproducción en cualquier medio, sin restricciones siempre que sin fines comerciales y que el trabajo original sea debidamente citado.
Resumen: En este trabajo se estudian los fenómenos metalúrgicos que ocurren en la soldadura SMAW de un acero inoxidable ferrítico AISI 430 con un acero inoxidable austenítico AISI 316L. Para el estudio se utilizaron dos tipos de electrodos: austenítico AWS E309L y dúplex AWS E2209-16, ambos con un diámetro de 3,2 mm. Las uniones soldadas se realizaron con un solo pase y se variaron simultáneamente la corriente y la velocidad de soldadura; las condiciones fueron $49 \mathrm{~A}$ y $2,4 \mathrm{~mm} \cdot \mathrm{s}^{-1}$ como valores bajos y $107 \mathrm{~A}$ y $4,3 \mathrm{~mm} \cdot \mathrm{s}^{-1}$ como valores altos. Se evaluó la influencia del tipo de electrodo y de los parámetros de soldadura en la evolución microestructural de las zonas afectadas por el calor y de las zonas de fusión, encontrando diferencias en la morfología y cantidad de ferrita delta para todas las condiciones estudiadas. Se evidenció crecimiento y refinación de grano ferrítico y formación de martensita en la zona afectada por el calor del metal base ferrítico. Se evaluó también la resistencia a la tensión hallando similitudes en todas las soldaduras.

Palabras-clave: Soldadura de aceros inoxidables; SMAW; Metalurgia de la soldadura; Acero inoxidable austenítico; Acero inoxidable ferrítico.

\section{Microstructural Transformations in Dissimilar Welds between Austenitic Stainless Steel and Ferritic Stainless Steel}

\begin{abstract}
This research studies the metallurgical transformations taking place the SMAW welding of AISI 316L austenitic stainless steel with AISI 430 ferritic stainless steel. To perform the study were used two different electrodes, AWS E309L austenitic and AWS E2209-16 duplex stainless steels $3.2 \mathrm{~mm}$ diameter. The joints were made with a single-pass welding including current and welding speed variations; the low values were $49 \mathrm{~A}$ and 2,4 mm. $\mathrm{s}^{-1}$ and the high values were $107 \mathrm{~A}$ and $4,3 \mathrm{~mm} \cdot \mathrm{s}^{-1}$. This study evaluated the influence of the type of electrode and the welding parameters on the microstructural evolution of heat affected and fusion zones. Also, differences were found on morphology and delta ferrite amount for all weld metals. The heat affected zone of the ferritic side showed grain coarsening and grain refinement with martensite at the grain boundaries. Tensile strength was similar for all welded joints.
\end{abstract}

Key-words: Stainless steel welding; SMAW; Welding metallurgy; Austenitic stainless steel; Ferritic stainless steel.

\section{Introducción}

Los aceros inoxidables (AI) son ampliamente usados en aplicaciones a elevadas temperaturas cuando los aceros al carbono y los aceros de baja aleación no presentan una adecuada resistencia a la corrosión. Los aceros inoxidables austeníticos (AIA) tienen mejor resistencia a la corrosión que los aceros inoxidables ferríticos (AIF) y los aceros inoxidables martensíticos por lo que son muy utilizados en industrias como la alimenticia, química y petroquímica. Sin embargo, los AIA presentan problemas de agrietamiento en caliente y de corrosión bajo tensión [1-3]. En vista del menor contenido de aleantes y del menor costo de los AIF, éstos son una opción viable ya que presentan una resistencia a la oxidación, para aplicaciones a altas temperaturas, comparable con la de los grados austeníticos, además los AIF pueden ser más resistentes al ataque del metal líquido que los AIA, por lo que son utilizados para algunas aplicaciones en las industrias del plomo y el cobre y en aplicaciones que involucran ciclos térmicos por su mayor conductividad térmica y su menor coeficiente de expansión térmica [2-4]. 
Existen aplicaciones de los aceros inoxidables en las cuales se requiere una transición en propiedades, como codos de tuberías para transporte de hidrocarburos o zonas de transición térmica en el proceso de refinación del cobre, por lo que se hace necesario realizar soldaduras disímiles de aceros inoxidables [5-7]. Los metales sometidos a procesos de soldadura experimentan cambios en su microestructura que dependen de los ciclos térmicos a los cuales fueron sometidos, de las propiedades físicas de cada material y de la composición química de éstos [8-10]. Los AIF presentan baja soldabilidad, crecimiento de grano y formación de fases frágiles como martensita o fase sigma, los AIA presentan buena soldabilidad pero pueden formar precipitados, comúnmente del tipo $\mathrm{M}_{23} \mathrm{C}_{6}$ que generan corrosión intragranular [1,11-13].

Para las soldaduras de Al los electrodos austeníticos son comúnmente utilizados pero su estructura final no es completamente austenítica sino que tienen un porcentaje de ferrita $\delta$, se ha determinado que cantidades de ferrita $\delta$ mayores al $5 \%$ disminuyen la susceptibilidad al agrietamiento en caliente de los AIA $[1,11,14]$. Los AIA presentan cuatro modos de solidificación que han sido estudiados desde la década del 80 principalmente por David et al. [15,16], Lippold y Savage [17] y Olson [18] por lo que es posible establecer una relación entre la cantidad y morfología de la ferrita $\delta$ y las propiedades mecánicas y de resistencia a la corrosión de las uniones. Los modos de solidificación se dividen en tipo A donde la transformación es completamente austenítica, tipo AF donde la transformación se da inicialmente como austenita y posteriormente se inicia la transformación ferrítica obteniendo una estructura de ferrita $\delta$ interdendrítica, el tipo FA en el cual la solidificación inicia como ferrita $\delta$ y posteriormente la austenita consume a la ferrita $\delta$ por medio de una reacción de difusión limitada. Este tipo es el más común para los AIA y produce morfologías de ferrita $\delta$ vermicular o en listones. Finalmente está el tipo $\mathrm{F}$ caracterizado por una solidificación completa como ferrita $\delta$ y una posterior transformación ferrita $\delta$-austenita en estado sólido, éste modo de solidificación es característico de los aceros inoxidables dúplex y presenta morfologías de ferrita $\delta$ acicular, globular o placas de austenita Widmanstätten $[1,11,14,15,19]$. Se han realizado uniones disímiles de AIA con AIF mediante soldadura por fricción [20,21] y soldadura por láser [22], estos procesos involucran cambios microestructurales diferentes a los de soldadura por arco eléctrico. En la industria petroquímica se emplea comúnmente la soldadura SMAW para reparaciones y debido a su fácil aplicación en campo, sin embargo, no se han desarrollado trabajos enfocados hacia el estudio de la influencia de este proceso en uniones disímiles de AIA con AIF, por lo tanto se hace necesario el entendimiento de la influencia de los parámetros de soldadura y la composición química de los electrodos en los cambios microestructurales que ocurren en la unión soldada disímil y la relación de éstos con las propiedades mecánicas del cupón de soldadura.

\section{Materiales y Métodos}

Se realizaron uniones soldadas disímiles mediante el proceso SMAW a tope con bisel en "V". Los metales base utilizados fueron acero inoxidable austenítico AISI 316L y acero inoxidable ferrítico AISI 430 comerciales, de $4 \mathrm{~mm}$ de espesor, y tamaños de $200 \mathrm{~mm} \times 100 \mathrm{~mm}$. Se utilizaron dos electrodos diferentes; AWS E2209-16 y E309L, de 3,2 mm diámetro. La unión se realizó con un solo pase y con dos condiciones de corriente y velocidad de soldadura; bajas 49 A y $2,4 \mathrm{~mm} \cdot \mathrm{s}^{-1}$ y altas $107 \mathrm{~A}$ y $4,3 \mathrm{~mm} \cdot \mathrm{s}^{-1}$.

La composición química de los metales de soldadura fue obtenida de la ficha técnica proporcionada por los proveedores, y la composición química de los metales base fue determinada mediante espectrometría de emisión óptica, éstas se presentan en la Tabla 1. Los parámetros controlados fueron: la corriente, la fuerza del arco y la velocidad de aplicación de la soldadura, los cálculos de entrada de calor se realizaron teniendo en cuenta un voltaje de $22 \mathrm{~V}$ y un factor de eficiencia de 0,75. En la Tabla 2 se presenta un promedio de los parámetros empleados en tres soldaduras para cada una de las cuatro condiciones a evaluar.

Los metales base y las uniones soldadas se caracterizaron microestructuralmente en la sección transversal por medio de microscopía óptica y de microscopía electrónica de barrido, las probetas se atacaron por inmersión en agua regia diluida, el pulido final se realizó con paño para acabado fino y alúmina de 0,3 $\mu \mathrm{m}$.

Se obtuvieron probetas para los ensayos de resistencia a la tensión en sentido transversal al cordón de soldadura de acuerdo con la norma ASTM E8, se emplearon probetas de $2 \mathrm{~mm}$ de espesor, las mediciones de microdureza Vickers se realizaron con una carga de $500 \mathrm{~g}$ y $15 \mathrm{~s}$ de sostenimiento, desde el metal base ferrítico hasta el metal base austenítico, cada 0,65 $\mathrm{mm}$. 
Tabla 1. Composición química de los metales base y metales de aporte.

\begin{tabular}{|c|c|c|c|c|c|}
\hline Acero inoxidable & $\begin{array}{c}C \\
(\% w)\end{array}$ & $\begin{array}{c}\mathrm{Cr} \\
(\% w)\end{array}$ & $\begin{array}{c}\mathrm{Ni} \\
(\% w)\end{array}$ & $\begin{array}{c}\text { Si } \\
(\% w)\end{array}$ & $\begin{array}{c}\text { Mn } \\
(\% w)\end{array}$ \\
\hline AISI 430 & 0,03 & 16,66 & 0,15 & 0,49 & 0,35 \\
\hline AISI 316L & 0,01 & 16,84 & 10,00 & 0,56 & 1,35 \\
\hline E309L & 0,03 & 23,70 & 13,70 & 0,80 & 0,85 \\
\hline E2209-16 & 0,03 & 22,80 & 9,30 & 0,73 & 0,68 \\
\hline Acero inoxidable & $\begin{array}{c}C u \\
(\% w)\end{array}$ & $\begin{array}{c}\mathrm{Ti} \\
(\% \mathrm{w})\end{array}$ & $\begin{array}{c}\text { Mo } \\
\text { (\%w) }\end{array}$ & $\begin{array}{c}\mathbf{P} \\
(\% w)\end{array}$ & $\begin{array}{c}N \\
(\% w)\end{array}$ \\
\hline AISI 430 & 0,10 & 0,01 & - & 0,03 & - \\
\hline AISI 316L & 0,10 & 0,01 & 2,02 & 0,04 & - \\
\hline E309L & 0,06 & - & 0,04 & 0,03 & - \\
\hline E2209-16 & 0,11 & - & 3,10 & 0,02 & 0,20 \\
\hline
\end{tabular}

Tabla 2. Parámetros de soldadura para la obtención de baja entrada de calor.

\begin{tabular}{ccccc}
\hline Condición & Electrodo & $\mathbf{v}(\mathbf{m m} / \mathbf{s})$ & I (A) & HI (J/mm) \\
CB & AWS E 309L & 2,2 & 49,8 & 372,0 \\
& AWS E 2209-16 & 2,6 & 48,8 & 311,0 \\
\multirow{2}{*}{ CA } & AWS E 309L & 3,9 & 106,1 & 453,0 \\
& AWS E 2209-16 & 4,7 & 108,7 & 387,0 \\
\hline
\end{tabular}

La cantidad de ferrita $\delta$ en el depósito de soldadura se predijo utilizando los diagramas de Schaeffler y WRC-92. Además, se midió con un ferritoscopio marca Fischer MP30E S y empleando cuantificación de fases por análisis de imagen con el analizador NIS elements de Nikon.

\section{Resultados y Discusión}

\subsection{Caracterización microestructural}

Las convenciones que se utilizarán en este artículo para hacer referencia a las diferentes zonas de la unión soldada son: MBF significa metal base ferrítico, MBA metal base austenítico, LF-F es el límite de fusión del lado del acero inoxidable ferrítico, LF-A es el límite de fusión del lado del acero inoxidable austenítico, ZAT-F es la zona afectada térmicamente del lado del acero inoxidable ferrítico, ZAT-A es la zona afectada térmicamente del lado del acero inoxidable austenítico, ZFA es la zona de fusión con el metal de aporte austenítico y ZFD es la zona de fusión con el metal de aporte dúplex. CB significa condiciones de baja corriente y baja velocidad de soldadura, CA quiere decir condiciones de alta corriente y alta velocidad de soldadura.

En la caracterización microestructural de los metales base se observó que el MBA contiene granos de austenita con maclas y caminos de ferrita $\delta$ orientados en la dirección de laminación. El MBF, por su parte, está compuesto por granos de ferrita delta y algunos precipitados dispersos en la matriz ferrítica, que podrían ser TiN, según lo reportado por Cleiton C. Silva et al. y E. Bayraktar et al. [7,23].

El perfil microestructural de los cupones soldados se presenta en la Figura 1, donde se distingue en la ZAT-F una zona de grano ferrítico grueso, seguida de una zona de grano recristalizado, ambas con martensita en los límites de grano ferrítico. Se observa que las probetas obtenidas con CA, para ambos electrodos, presentan una ZAT-F de mayor extensión que las soldaduras realizadas con $\mathrm{CB}$, dicha zona se delimita por la martensita que oscurece los contornos de grano y se señala con una línea blanca punteada en la Figura 1. Las cuatro condiciones soldadas presentaron zonas afectadas térmicamente con microestructuras similares que se describen a continuación.

La microestructura de ZAT-F es caracterizada por una zona de grano grueso, en la cual, el desplazamiento de los límites de grano ferríticos provoca que los granos grandes "consuman" a los granos pequeños por el aporte energético inherente al proceso de soldadura [9], dicha zona está seguida por una zona de grano recristalizado, 
proveniente del proceso de recuperación y nucleación de nuevos granos además del ascenso y aniquilación de dislocaciones generadas durante el proceso de fabricación del metal base [24]. En toda la ZAT-F se obtuvo martensita intragranular y unas pequeñas cantidades martensita intergranular, esta fase proviene de la transformación de la austenita formada a alta temperatura y su posterior enfriamiento $[1,11]$.

La ZAT-A presentó una microestructura compuesta por granos de austenita con maclas sin variación perceptible de tamaño para CB y con un pequeño aumento de tamaño de grano austenítico en la zona cercana a la raíz de las soldaduras al emplear $C A$, se formó ferrita $\delta$ en los límites de grano austenítico, condición que se extendió alrededor de dos granos austeníticos desde la línea de fusión hacia el MBA, la formación de ferrita $\delta$ en los límites de grano restringe el crecimiento de grano austenítico y disminuye la susceptibilidad al agrietamiento en caliente [1,11,25-27].

La microestructura de los límites de fusión y la zona afectada térmicamente fue similar para todas las condiciones evaluadas y estas se muestran en la Figura 2. El LF-A está caracterizado por dendritas de ferrita $\delta$ provenientes de metal fundido, presentes en los límites de grano austenítico del MBA. El LF-F muestra un crecimiento epitaxial de las dendritas del metal de soldadura que parten de los granos del MBF.

Las fases presentes en todos los depósitos de soldadura obtenidos fueron austenita y ferrita $\delta$, su morfología y proporción está determinada por la composición química del electrodo y las velocidades de enfriamiento [17,26], por lo que al emplear dos electrodos con diferente composición y dos condiciones de corriente y velocidad de soldadura se generaron zonas de fusión con diferencias microestructurales que se detallan a continuación.

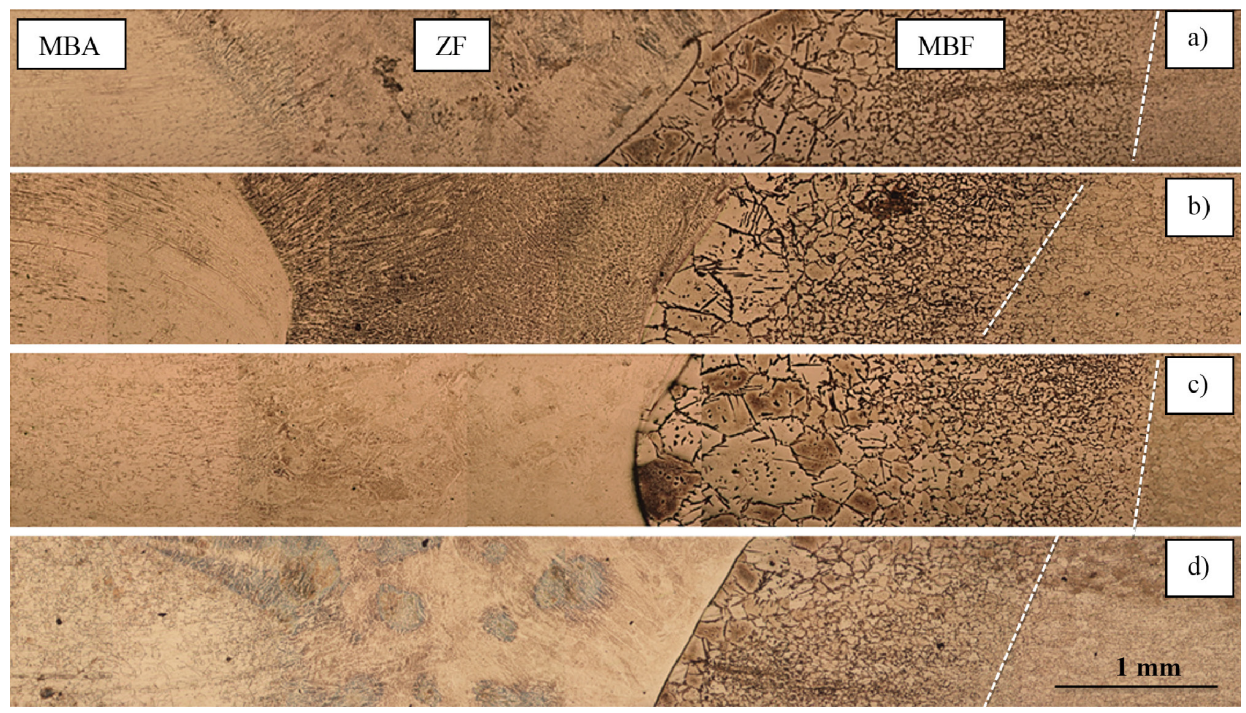

Figura 1. Microestructura de las soldaduras SMAW AISI 316L - AISI 430. a) aporte E309L CA; b) aporte E309L CB; c) aporte E2209-16 CA y d) aporte E2209-16 CB.
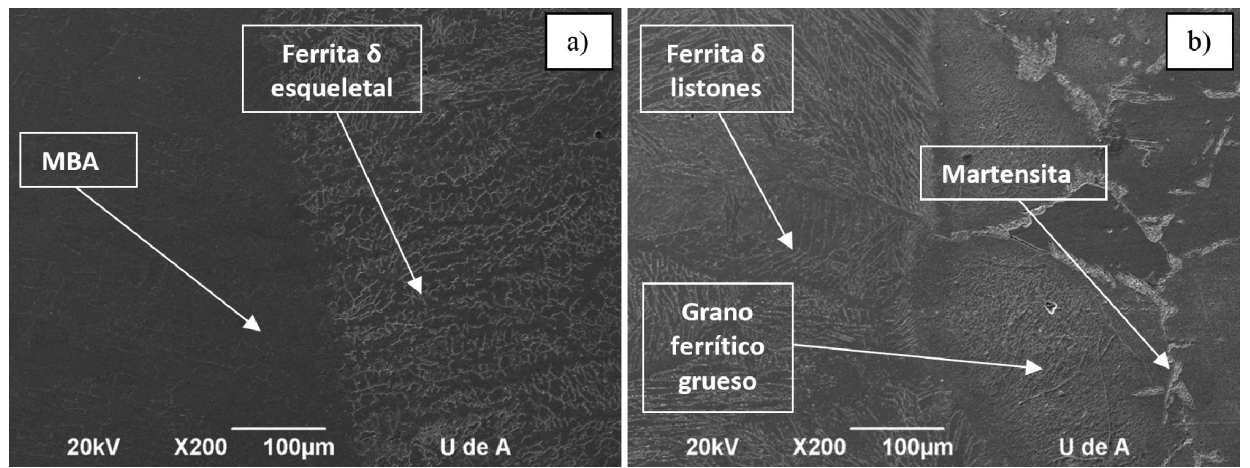

Figura 2. Microestructura de los LF de la soldadura realizada con el E309L CA. a) LF-A; b) LF-F. 
La microestructura obtenida en la ZFA está compuesta por una matriz austenítica con ferrita $\delta$ con dos morfologías diferentes, vermicular y en listones, estas estructuras son características del modo de solidificación FA, es decir, ferrita-austenita. En el rango FA la solidificación inicia con la precipitación de ferrita primaria seguida de la formación de austenita en la celda ferrítica y el límite de la dendrita, al avanzar el enfriamiento, la ferrita se hace más inestable y la austenita consume a la ferrita por medio de una reacción de difusión controlada. La morfología esqueletal o vermicular se presenta debido a velocidades de enfriamiento moderadas y relaciones $\mathrm{Cr}$ eq $/ \mathrm{Ni}_{\text {eq }}$ bajas, la morfología en listones se presenta cuando las velocidades de enfriamiento son altas y la difusión durante la transformación ferrita-austenita es restringida, donde se promueve la transformación en forma de listones poco espaciados que genera un patrón de ferrita residual a través de la dendrita original [1,16,17,29].

En la ZFA de la soldadura realizada con CB se observa una matriz austenítica con dendritas esqueletales de ferrita $\delta$, fase oscura, que se muestra en la Figura $3 a$. En el caso de la ZFA de la soldadura realizada con CA, que se presenta en la Figura $3 b$, se evidencia crecimiento dendrítico con ferrita $\delta$ vermicular y en listones, con morfología predominante en listones. En la ZF, cerca del MBA se observa una morfología de ferrita $\delta$ vermicular, mientras que en la ZF cerca del lado del MBF se presenta una ferrita $\delta$ en listones, estas diferencias se producen por los distintos ciclos térmicos que experimenta la soldadura en cada punto $[1,9,10]$, además de las diferencias en propiedades térmicas que presentan los metales base en las soldaduras disímiles realizadas, una menor conductividad térmica MBA frente al MBF [30].

En las Figuras $3 c$ y $3 \mathrm{~d}$ se muestra la ZFD caracterizada por la presencia de tres tipos de microestructura, fenómeno común en el depósito de soldadura donde cada punto de la zona de fusión experimenta ciclos térmicos diferentes y por lo tanto velocidades de enfriamiento distintas. A pesar de que la ZFD presenta similitudes con CB y CA, cabe anotar que el tamaño de grano ferrítico previo formado durante la solidificación, es de mayor tamaño en las probetas soldadas con CA que con CB. Las microestucturas presentes en el depósito dúplex son:
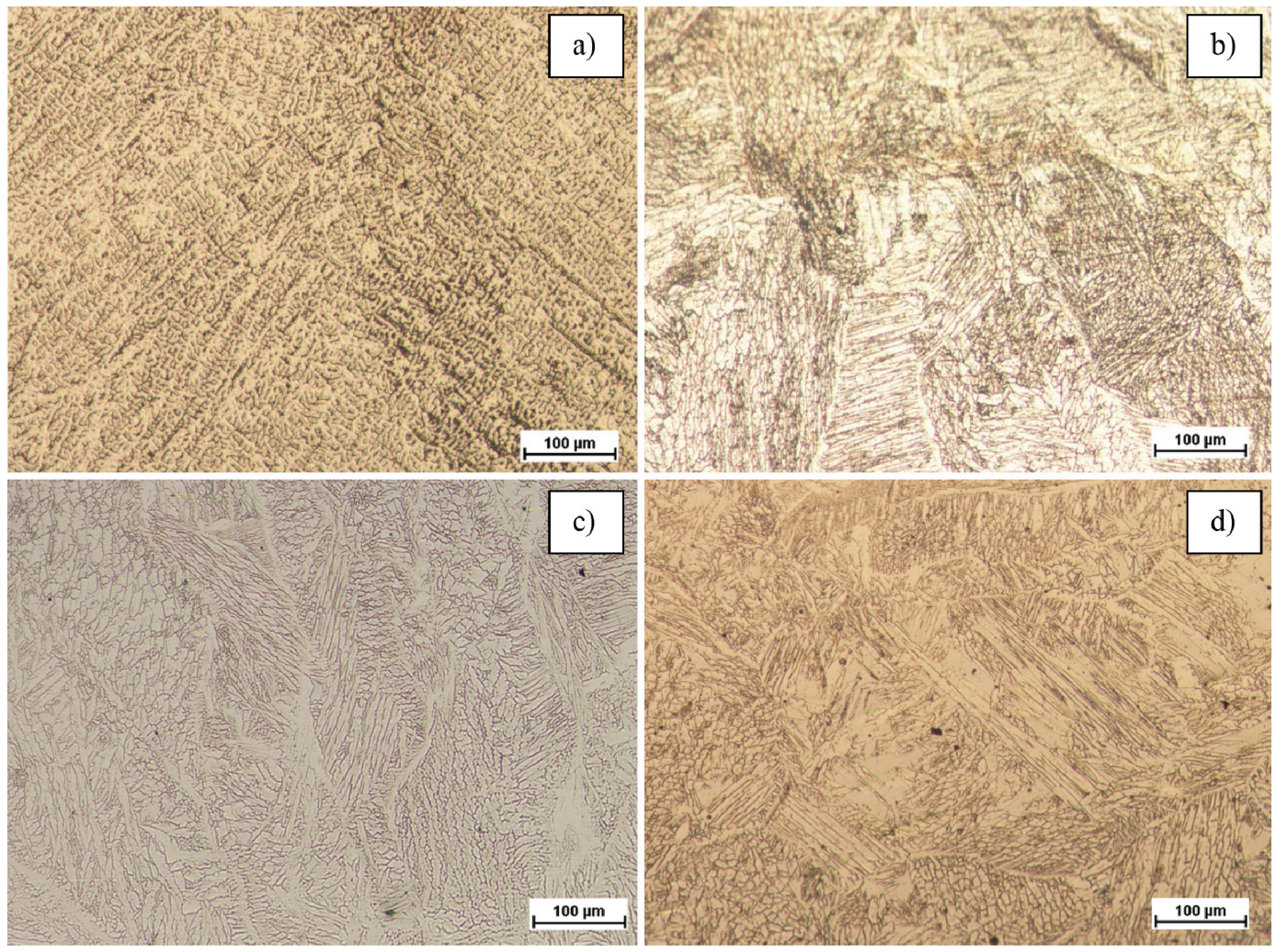

Figura 3. Microestructura de las ZF. a) ZFA CB); b) ZFA CA; c) ZFD CB y d) ZFD CA. 
1. Ferrita y austenita aciculares dentro de los granos previos de ferrita formados durante la solidificación y una red continua de austenita en los límites de grano de esta ferrita: Este mecanismo de solidificación se caracteriza por una microestructura completamente ferrítica al final de la solidificación, luego de que el metal se enfría por debajo de la línea de solvus de la ferrita, inicia la transformación en estado sólido de ferrita a austenita, la cantidad de austenita formada depende principalmente de las relaciones $\mathrm{Cr}_{\text {eq }} / \mathrm{Ni}_{\text {eq }}$. La austenita se forma inicialmente en los límites de grano ferrítico, luego el frente de transformación rompe formando agujas de austenita dentro de la ferrita, produciendo una estructura acicular parecida a los listones de ferrita $\delta$ formados en el modo de solidificación FA, esta transformación es similar a una transformación martensítica o por desplazamiento ya que como el intervalo de temperaturas entre la línea de solidus y la línea de solvus de la ferrita es tan grande, se suavizan los gradientes composicionales por la difusión de Cromo y Níquel en esta zona generando listones de ferrita y austenita con composición similar [1,16,17,19].

2. Ferrita globular, la cual se genera por inestabilidades térmicas de los otros tipos de ferrita, principalmente la acicular y se caracteriza por ferrita delta en forma de glóbulos distribuidos aleatoriamente en una matriz de austenita [16].

3. Ferrita y austenita Widmanstätten dentro de los granos previos de ferrita. La solidificación se da completamente como ferrita, el inicio de la transformación ferrita-austenita ocurre en los límites de grano ferrítico, posterior a eso se nuclea la austenita en el límite de grano y se forman placas de austenita Widmanstätten, como ha sido referenciado, esta transformación no es masiva sino que obedece a una transformación difusiva [1,17]. Debe notarse que los granos de ferrita formados inicialmente en la ZFD con CA son de mayor tamaño que los formados en la ZFD con CB $[1,15,17,19]$.

\subsection{Resistencia a la tensión, dureza y cantidad de ferrita delta $\delta$}

En la Tabla 3 se presentan los resultados de los ensayos de tensión, se evidencia que no existe una diferencia significativa en las propiedades tensiles de las uniones soldadas para las cuatro condiciones evaluadas y son comparables con las propiedades del AISI 430 [30]. En todos los casos la falla ocurrió en el MBF lejos de la ZAT mostrando un comportamiento dúctil similar al del acero inoxidable ferrítico AISI 430 [30]. Estos resultados indican que el crecimiento de grano ferrítico y la formación de martensita generados en la ZAT-F, no fueron factores determinantes en la falla bajo tensión de las probetas sometidas al ensayo.

En la Tabla 4 se presentan los resultados de la cantidad de ferrita $\delta$ en porcentaje y número de ferrita (FN), medidos con el ferritoscopio y con el analizador de imágenes y los calculados a través de los diagramas de Schaeffler y WRC-92. Para la predicción de la cantidad de ferrita $\delta$ mediante diagramas constitucionales se midieron los porcentajes de dilución: $20 \%$ de dilución con el electrodo dúplex y $23 \%$ de dilución con el electrodo austenítico. Se observa de la Tabla 4 que al aumentar la entrada de calor, aumenta la cantidad de ferrita delta. Se observa también que los valores medidos y los valores calculados difieren mucho unos de otros, las posibles causas de dichas diferencias se describen a continuación.

Tabla 3. Resultados de los ensayos de resistencia a la tensión.

\begin{tabular}{cccc}
\hline Electrodo y condición & Resistencia última (MPa) & Límite de cedencia (MPa) & Elongación (\%) \\
AWS E309L CB & 485 & 378 & 34 \\
AWS E309L CA & 499 & 387 & 34 \\
AWS E2209-16 CB & 505 & 361 & 36 \\
AWS E2209-16 CA & 488 & 369 & 32 \\
\hline
\end{tabular}

Tabla 4. Cantidad de ferrita delta medida y calculada en la zona de fusión.

\begin{tabular}{ccccc}
\hline $\begin{array}{c}\text { Electrodo y } \\
\text { condición }\end{array}$ & $\begin{array}{c}\text { \% ferrita } \boldsymbol{\delta} \\
\text { (ferritoscopio) }\end{array}$ & $\begin{array}{c}\text { \% ferrita } \boldsymbol{\delta} \text { (analizador } \\
\text { de imágenes) }\end{array}$ & $\begin{array}{c}\text { \% ferrita } \boldsymbol{\delta} \\
\text { (Schaeffler) }\end{array}$ & $\begin{array}{c}\text { FN } \\
\text { (WRC 1992) }\end{array}$ \\
AWS E309L CB & $9 \pm 2$ & $25 \pm 3$ & 12 & 16 \\
AWS E309L CA & $14 \pm 3$ & $30 \pm 3$ & & 27 \\
AWS E2209-16 CB & $25 \pm 3$ & $41 \pm 3$ & 47 & 27 \\
AWS E2209-16 CA & $31 \pm 2$ & $42 \pm 3$ & & \\
\hline
\end{tabular}




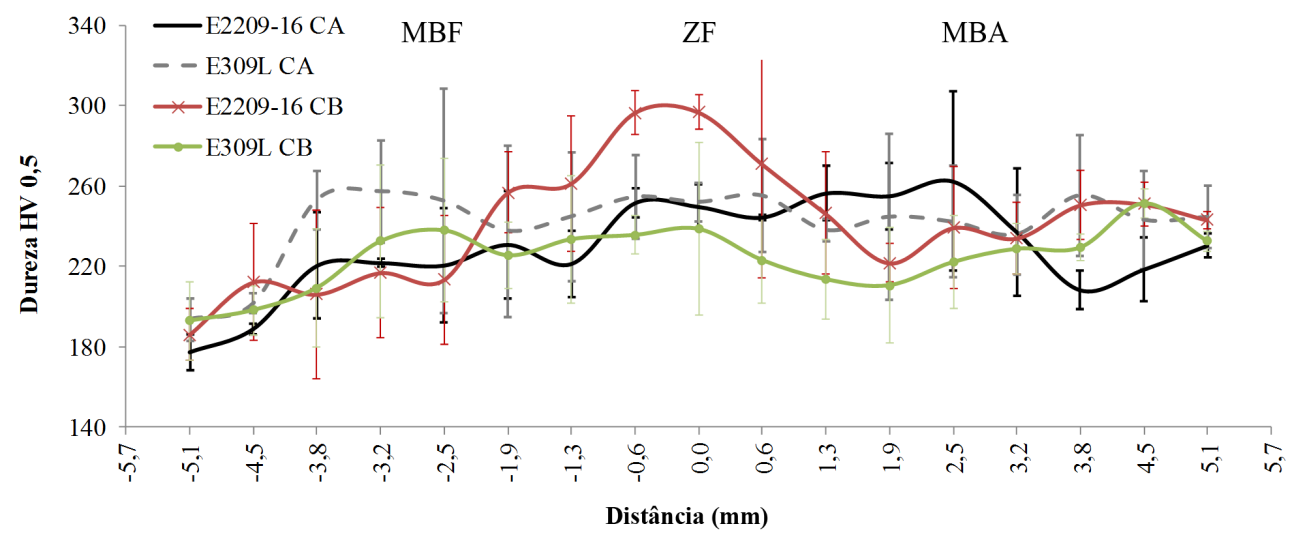

Figura 4. Perfiles de microdureza.

En la determinación del contenido de ferrita $\delta$, se ha reportado que para un mismo cordón de soldadura se obtienen variaciones locales en la cantidad de ferrita $\delta$ [16,17,31,32], debido a microsegregaciones en composición producidas por diferentes ciclos de enfriamiento o a la pérdida de elementos durante el proceso de soldadura, por lo que la cantidad de ferrita $\delta$ del metal de soldadura debe obtenerse de varias mediciones como lo indica la norma DIN 32514 [33]. Los métodos predictivos como los diagramas de Schaeffler y WRC-92 no toman en cuenta las velocidades de enfriamiento que son un factor determinante en la cantidad de ferrita $\delta$ presente en los depósitos de soldadura [15-19,28,31,32,34-37], además, el diagrama de Schaeffler no tiene en cuenta el contenido de Nitrógeno en su cálculo del Níquel equivalente, que para el electrodo dúplex es de $0,2 \%$. La técnica de permeabilidad magnética (Ferritoscopio Fischer) presenta algunas limitaciones prácticas relacionadas con las dimensiones de la muestra, por ejemplo, al usar muestras de tamaño reducido pueden disminuir los valores de ferrita $\delta$ medidos debido al efecto de borde. En cuanto a la determinación metalográfica, los errores en la medición del porcentaje en volumen de ferrita $\delta$ dependen de la selección correcta del reactivo de ataque, la habilidad del operario para distinguir entre la ferrita $\delta$ y otras fases como carburos, nitruros o fase sigma, por lo que con esta técnica tiende a sobreestimarse la cantidad de ferrita $\delta$. Asunción Valiente [37] no recomienda esta técnica para depósitos de soldadura debido a la morfología irregular y delgada de la ferrita $\delta[31,37]$.

Es importante anotar que el valor de ferrita $\delta$ calculado con el diagrama WRC-92 se reporta en FN y no es comparable con el porcentaje de ferrita hallado con otros métodos. Autores como Shinozaki et al. [38], Lilijas y Qvartof [39] y Gill et al. [40], reportan relaciones entre el FN y el porcentaje de ferrita, y aunque concuerdan que FN exagera el porcentaje de ferrita, no coinciden con la relación entre los dos [34].

En la Figura 4 se presentan los perfiles de microdureza obtenidos para las condiciones soldadas, es de resaltar que la dureza de ZFD CB fue en promedio $296 \mathrm{HV}$, valor que sobrepasa la dureza medida para las demás condiciones soldadas, concordando con algunos autores [6,41,42] con que la cantidad de ferrita $\delta$ no es el factor más determinante en la dureza de los depósitos de soldadura, por lo que podría atribuirse esta mayor dureza a la morfología acicular que predomina en esta condición de soldadura [43].

\section{Conclusiones}

Con base en los resultados experimentales para la unión de acero inoxidable austenítico con acero inoxidable ferrítico utilizando metales de aporte austenítico y dúplex, con baja y alta corriente y velocidad de soldadura puede concluirse que:

En la microestructura, para las condiciones evaluadas, la principal diferencia se encontró en la zona de fusión, donde las soldaduras realizadas con el metal de aporte austenítico presentaron una morfología de ferrita delta vermicular en las soldaduras realizadas con CB y morfología predominante en listones para las de CA. En el caso del depósito dúplex, se observó que en ambas condiciones de corriente y velocidad de soldadura, las microestructuras consisten de una combinación de ferrita $\delta$ acicular y en glóbulos, y austenita Widmanstätten, donde la morfología acicular fue predominante para el depósito obtenido a CB y la morfología Widmanstätten fue predominante para 
las soldaduras obtenidas con CA. En la ZAT-A, muy próximo a la LF-A, se evidenció la presencia de formación de ferrita $\delta$ en los límites de grano austenítico. Mientras que en la ZAT-F, la microestructura consiste de una zona de grano ferrítico grueso y una zona de grano ferrítico recristalizado, con martensita en los límites de grano ferríticos.

En relación con las propiedades mecánicas evaluadas, los dos materiales de aporte se comportaron satisfactoriamente, las propiedades tensiles en todos los casos fueron similares. Los cambios en el tamaño de grano ferrítico, la formación de martensita, la precipitación de fases y la variación en microdureza no afectaron la resistencia última a la tensión, el porcentaje de elongación y el límite de cedencia de las uniones soldadas.

Los porcentajes de ferrita $\delta$ medidos con el ferritoscopio y el analizador de imágenes, y los calculados con los diagramas de Schaeffler y WRC-92 difieren. Se recomienda adoptar el FN para referirse a cantidades de ferrita $\delta$ ya que el porcentaje de ferrita $\delta$ en volumen presenta más inexactitudes.

El electrodo austenítico AWS E309L representa una opción más económica y con similar desempeño mecánico que el electrodo dúplex AWS E2209-16 para realizar la soldadura disímil de AISI 430 y AISI 316L.

\section{Agradecimientos}

Los autores expresan sus agradecimientos al CODI de la Universidad de Antioquia por el apoyo financiero a través del proyecto MDC10-1-07 y de la estrategia de sostenibilidad 2014-2015, y a Colciencias por la beca Joven Investigador.

\section{Referencias}

[1] Lippold JC, Kotecki DJ. Welding metallurgy and weldability of stainless steels. USA: Jhon Wiley and Sons; 2005. 357 p.

[2] Duarte P, Corrêa EO, Barbedo ND, Souza PO, Gonçalves JL, Diacenco AA. Mechanical and microstrutural characterization of weldments of ferritic stainless steel AISI 444 using austenitic stainless steels filler metals. Journal of ASTM International. 2012;9(2):1-9.

[3] Kumaragurubaran B, Shanmugam K, Balasubramanian V, Senthilkumar T. High temparture tensile properties of shielded metal arc welded ferrtiic stainless Steel, India. International Journal of Material Science. 2008;3(3):229-237.

[4] Olson DL, Siewert TA, Liu S, Edwards GR. ASM Handbook, Volume 6: welding, brazing and soldering. Materials Park: ASM International; 1993.

[5] Samal MK, Seidenfuss M, Roos E, Balani K. Investigation of failure behavior of ferritic-austenitic type of dissimilar steel welded joints. Engineering Failure Analysis. 2011;18(3):9991008. http://dx.doi.org/10.1016/j.engfailanal.2010.12.011.

[6] Kaçar R, Baylan O. An investigation of microstructure/property relationships in dissimilar welds between martensitic and austenitic stainless steels. Materials \& Design. 2004;25(4):317329. http://dx.doi.org/10.1016/j.matdes.2003.10.010.

[7] Silva CC, Miranda HC, de Sant'Ana HB, Farias JP. Austenitic and ferritic stainless steel dissimilar weld metal evaluation for the applications as-coating in the petroleum processing equipment. Materials \& Design. 2013;47:1-8. http://dx.doi.org/10.1016/j. matdes.2012.11.048

[8] Granjon H. Fundamentals of welding metallurgy. England: Woodhead Publishing; 1991. 210 p.. http://dx.doi.org/10.1533/9781845698805.

[9] Easterling K. Introduction to the physical metallurgy of welding. UK: Butterworths and Co Ltd; 1983. 141 p.

[10] Grong O. Metallurgical modelling of welding. London: The Institute of Materials; 1994. 532 p.

[11] Folkhard E. Welding metallurgy of stainless steels. New York: Springer- Verlag Wien; 1998. 279 p.

[12] Lakshminarayanan AK, Shanmugam K, Balasubramanian V. Effect of welding processes on tensile and impact properties, hardness and microstructure of AISI 409M ferritic stainless joints fabricated by duplex stainless steel filler metal. Journal of Iron and Steel Research International. 2009;16(5):66-72. http://dx.doi.org/10.1016/S1006-706X(10)60013-1.

[13] Silva CC, Farias JP, Miranda HC, Guimarães RF, Menezes JWA, Neto MAM. Microstructural characterization of the HAZ in AISI 444 ferritic stainless steel welds. Materials Characterization. 2008;59(5):528533. http://dx.doi.org/10.1016/j.matchar.2007.03.011.

[14] Ku S. Welding metallurgy. 2. ed. USA: John Wiley \& Sons; 2003. $224 \mathrm{p}$.

[15] David SA, Goodwin GM, Braski DN. Solidification behavior of austenitic stainless steel filler metals. Welding Journal. 1978;58(11):330s-336s.

[16] David, SA. Ferrite morphology and variations in ferrite content in austenitic stainless steel welds. Welding Journal. 1981;60(4):63s-71s.

[17] Lippold JC, Savage WF. Solidification of austenitic stainless steel weldments: Part 2-The effect of alloy composition on ferrite morphology. Welding Journal. 1980;59(2):48s-58s.

[18] Olson DL. Prediction of austenitic weld metal microstructure and properties. Welding Journal. 1984;64(10):281-295.

[19] Kaçar R. Effect of solidification mode and morphology of microstructure on the hydrogen content of duplex stainless steel weld metal. Materials \& Design. 2004;25(1):1-9. http:// dx.doi.org/10.1016/S0261-3069(03)00169-9.

[20] Sathiya P, Aravindan S, Haq AN. Tensile properties of similar AISI 304 austenitic and AISI 430 ferritic stainless steels joined by friction welding. Multidiscipline Modeling in Materials and Structures. 2008;4(2):141-154. http://dx.doi. org/10.1163/157361108784050112.

[21] Manideep D, Balachanda K. Welding parameters-metallurgical properties correlation of friction welding of austenitic stainless steel and ferritic stainless steel. Journal of Applied Sciences. 2012;12(10):1013-1019. http://dx.doi.org/10.3923/ jas.2012.1013.1019.

[22] Khan MMA, Romoli L, Fiaschi M, Dini G, Sarri F. Laser beam welding of dissimilar stainless steels in a fillet joint configuration. 
Journal of Materials Processing Technology. 2012;212(4):856867. http://dx.doi.org/10.1016/j.jmatprotec.2011.11.011.

[23] Bayraktar E, Chevalier JP, Kaplan D, Devillers L. Physical understanding of ferrite grain growth during welding in interstitial free steels (IFS) +. Arabian Journal for Science and Engineering. 2009;34(1C):115-127.

[24] Phillips R. Cristalls, defects and microstructures: modelling across scales. UK: University of Cambridge; 2004. 780 p.

[25] Thomas RD Jr. HAZ Cracking in thick sections of austenitic stainless steels-Part II. Welding Journal. 1984;63(12):355-368.

[26] Lippold JC, Baeslack lii WA, Varol I. Heat-affected zone liquation cracking in austenitic and duplex stainless steels. Welding Journal. 1988;71(1):1-14.

[27] Shankar, V, Gill TPS, Mannan SL, Sundaresan S. Solidification cracking in austenitic stainless steel welds. 2003;28(3-4):359382

[28] Inoue H., Koseki T. Clarification of solidification behaviors in austenitic stainless steels based on welding process. Nippon Steel Technical Report. 2007;95:62-70.

[29] Madhusudhan Reddy G, Mohandas T, Sambasiva Rao A, Satyanarayana VV. Influence of welding processes on microstructure and mechanical properties of dissimilar austenitic-ferritic stainless steel welds Materials and manufacturing Processes. Materials and Manufacturing Processes. 2005;20(2):147-173. http://dx.doi.org/10.1081/AMP-200041844.

[30] Davis, JR. ASM Speciality handbook: stainless steels. Ohio: ASM International; 1994. v. 2, p. 489-490.

[31] Kotecki DJ. Ferrite determination in stainless steel welds: advances since 1974. Welding Journal. 1996;76(1):24-37.

[32] Prasad Rao K, Prasannakumar S. Assesment criterion for variability of delta ferrite in austenitic weld and clad metals. Welding Journal. 1984;63(7):231-236.

[33] German Institute for Standardization - DIN. DIN 32514-part 1. Determination of ferrite number of austenitic weld metalmeasure- ment method. Düsseldorf: DIN; 1990.
[34] David SA, Vitek JM, Hebble TL. Effect of rapid solidification on stainless steel weld metal microstructures and its implications on the Schaeffler diagram. Welding Journal. 1987;66(10):289300.

[35] Esmailian M. The effect of cooling rate and austenite grain size on the austenite to ferrite transformation temperature and different ferrite morphologies in microalloyed steels. Iranian Journal of Materials Science \& Engineering. 2010;7(1):7-14.

[36] Siewert TA, McCowan CN, Olson DL. Ferrite number prediction to $100 \mathrm{FN}$ in stainless steel weld metal. Welding Journal. 1988;67(12):289-298.

[37] Valiente, M. Predictive and measurement methods for delta ferrite determination in stainless steels. Welding research. 2012;91:113-121.

[38] Shinozaki L, Ke L, North TH. Hydrogen cracking in duplex stainless steel weld metal. Welding Journal. 1992;171(11):387s-396s.

[39] Liljas M, Qvarfort R. Influence of nitrogen on weldments in UNS S 31803. In: Proceedings of the Conference Duplex Stainless Steels 86; 1986; The Hauge, The Netherlands. The Netherlands: Nederlands Institut Voor Lastechniek; 1986. p. 244-256.

[40] Gill TPS, Dayal RK, Gnanamoorthy JB. Estimation of delta ferrite in austenitic stainless steel weldments by an electrochemical technique. Welding Journal. 1979;59(12):375s-378s.

[41] Zumelzu E, Sepúlveda J, Ibarra M. Influence of microstructure on the mechanical behaviour of welded 316 L SS joints. Journal of Materials Processing Technology. 1999;94(1):36-40. http:// dx.doi.org/10.1016/S0924-0136(98)00450-6.

[42] Muthupandi V, Bala Srinivasan P, Seshadri SK, Sundaresan S. Effect of weld metal chemistry and heat input on the structure and properties of duplex stainless steel welds. Materials Science and Engineering A. 2003;358(1-2):9-16. http://dx.doi. org/10.1016/S0921-5093(03)00077-7.

[43] Ku S. Welding metallurgy. 2. ed. USA: John Wiley \& Sons; 2003. $461 \mathrm{p}$. 